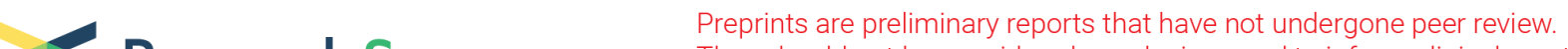

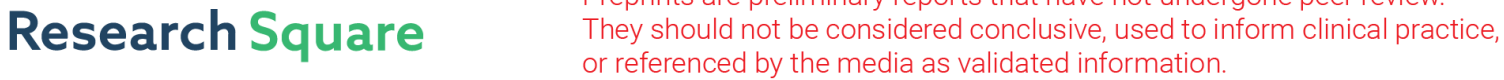 \\ The PCSK9 Involves in the Dyslipidemia of Ovariectomized Mice and Its Mechanism
}

\section{Dan Zhao}

Sichuan University West China School of Basic Medical Sciences and Forensic Medicine

\section{Xue-qin Zhang}

Sichuan University West China School of Basic Medical Scinences and Forensic Medicine

\section{Wen-jing Guo}

Sichuan University West China School of Basic Medical Sciences and Forensic Medicine

\section{Zhi-hui Cui}

Sichuan University West China School of Basic Medical Sciences and Forensic Medicine

\section{Yi-cheng Wang}

Sichuan University West China of Basic Medical Sciences and Forensic Medicine

\section{Yong-dan Ma}

Sichuan University West China School of Basic Medical and Forensic Medicine

\section{Li-xue Zhang}

Sichuan University West China School of Basic Medical Sciences and Forensic Medicine Jin-hu Zhang

DM Wayanad Institute of Medical Sciences Department of Forensic Medicine

\section{Li Nie}

Sichuan University West China School of Basic Medical Sciences and Forensic Medicine

\section{Limin Yue ( $\nabla$ yuelimin@scu.edu.cn )}

Sichuan University West China School of Basic Medical Sciences and Forensic Medicine https://orcid.org/0000-0002-7631-5733

\section{Research Article}

Keywords: Estrogen deficiency, PCSK9, Disorders of lipid metabolism, LDLR

Posted Date: October 22nd, 2021

DOl: https://doi.org/10.21203/rs.3.rs-939848/v1

License: (c) (i) This work is licensed under a Creative Commons Attribution 4.0 International License. Read Full License 


\section{Abstract}

Ovarian failure in postmenopausal female leads E2 to dramatic decrease which is an important reason of menopausal dyslipidemia. PCSK9 as a secretory lipid metabolic regulator plays a critical role in the cholesterol metabolism by negatively regulating LDLR in hepatocytes. Clinical data showed PCSK9 was elevated and positively correlated with LDL-C in the blood of postmenopausal women. However, the relationship between E2 and PCSK9 and the role of PCSK9 in postmenopausal dyslipidemia are still unclear. In this research, 10-week-old ovariectomized mice were fed for 4 weeks with normal diet or highfat diet, then tested the lipid metabolism profiles and PCSK9 in the blood and the expression of LDLR and PCSK9 in the liver. On this basis, PCSK9-/-ovariectomized mice were used to further verify the effect of PCSK9 in dyslipidemia of ovariectomized mice. Finally, the ovariectomized mice with high-fat diet were subcutaneous injected respectively with E2 or PCSK9 inhibitor alone or both together for 2 weeks and were tested as previous experiment. The results showed PCSK9, TC and LDL-C all increased in the blood of in WT ovariectomized mice and their PCSK9 is positively correlated with LDL-C, while there were on obvious lipid metabolism disorder in the $\mathrm{PCSKO}^{-/-}$ovariectomized mice. PCSK9 inhibitor increased the LDLR on the liver and ameliorated the dyslipidemia in WT ovariectomized mice. It suggests that PCSK9 plays an important role in the dyslipidemia of ovariectomized mice, which provides a new strategy for clinical diagnosis and treatment of the dyslipidemia in post-menopause.

\section{Introduction}

One of the main ovarian functions is the synthesis and secretion of female estrogen (E2) which maintains the structure and fuctions of reproductive system, also involved in regulating the functions of other systems and organs, such as cardiovascular system, urinary system, nervous system and so on[1]. Ovarian failure in menopausal women inevitablyleads to a sharp decrease of E2 which causes metabolism syndrome including obesity, cardiovascular disease, osteoporosis, anxiety etc. The disorders of lipid metabolism after menopause usually encompass total cholesterol (TC), low-density lipoprotein cholesterol (LDL-C) and triglycerides (TG) increased and high-density lipoprotein cholesterol (HDL-c) decreased [2], in which LDL-c is an important risk factor of atherosclerosis and cardiovascular disease[3]. In recent years, the consumption of a high-fat diet (HFD) has been increasingly viewed as a significant factor for aggravating the disorder of lipid metabolism in menopause[4]. However, the mechanism of lipid metabolism disorders caused by estrogen deficiency among the women in menopause is still unclear.

Low-density lipoprotein receptor (LDLR) on the hepatocyte membranes plays an important role in regulating LDL-c in blood. LDL-c binding to LDLR is internalized by endocytosis under the participation of reticulin, then LDL-c dissociates from LDLR for cellular utilization oris degraded in the acidic environmentof intracellular lysosomes, while LDLR is transported back to the cell membrane for recycle[5]. Once the LDLR fails to recycle, the number of LDLRs in the cell membrane decreases, the level of LDL-C in blood will elevate. 
Proprotein convertase subtilisin/kexin type-9 (PCSK9) is a secreted protein firstly found in the liver a few years ago. PCSK9 may binds to the LDLR on cell membranes to form a complex, which hinders its recycling to lead to its degradation[6] so that the level of LDL-c in blood incrase. Therefore, PCSK9 is considered to be a negative regulator of cholesterol metabolism and is related to abnormal lipid metabolism and coronary heart disease[7]. PCSK9 inhibitors have been used in the treatment of hypercholesterolemia and mixed dyslipidemia. Clinical data has suggested that the elevation of PCSK9 is positively correlated with the increase of blood LDL-C in postmenopausal women[7]. Thus, it is not difficult to infer that there is an inevitable link among estrogen deficiency and PCSK9, as well as the lipid metabolism disorders in postmenopausal women.

In this research, wild-type $(W T)$ C57BL/6 female mice and PCSK9 knockout $\triangle P C S K 9^{-/}$- ffemale mice were used to explore PCSK9 involves in the dyslipidemia of ovariectomized mice and its mechanism. At first, we fed the mice with high-fat diet or normal diet for 4 weeks after ovariectomy, then we tested the mouse lipid metabolism profiles,PCSK9 in the blood and the expression of LDLR in liver, analyzed the correlation between PCSK9 and the lipid profiles. Futhermore, we treated the high-fat diet fed ovariectomized mice respectivelywith estrogen, PCSK9 inhibitor alone or the combination of the both for 2 weeks and checked the same items as the above. The purpose is to provide a new cluefor the diagnosis, treatment and prevention of postmenopausal lipid metabolism disorders.

\section{Materials And Methods}

Animal Experiments

All animal experiments were approved by the Sichuan University academic ethics and ethics committee and conducted in strict accordance with laboratory animal care and use program.

10-week-old C57BL/6 female mice(SPF) were purchased from Chengdu Dashuo(SCXK (Chuan) 2015030). PCSK9-/- mice were generated by Beijing Biocytogen Co. Itd. Mice werein the condition of ambient temperature $23-25^{\circ} \mathrm{C}$, relative humidity $40 \%-60 \%$, light $12 \mathrm{~h} / \mathrm{d}$ and free drinking and eating.

To clarify the changes of PCSK9 and lipid metabolism, as well as the relationship between both of them in ovariectomized mice, 20 10-week-old C57BL/ 6 female mice were divided into ovariectmized group and sham operation group. The half of two proups were fed with normal diet for 4 weeks, and the others were fed with high-fat diet for 4 weeks. Then all of them were checked E2, TC, TG, LDL-C, HDL-c and PCSK9 in the blood also we measured the PCSK9 and LDLR in their liver tissues.

To further verify PCSK9 is a important cause of dyslipidemia in ovariectomized mice, 8 10-week-old wildtype female mice (WT) and 8 10-week-old PCSK9 ${ }^{-/-}$female mice were ovariectomized and fed with highfat diet for 4 weeks, then, they were tested the same items as the above.

Next experiment was done in 20 female 10-week-old C57BL/ 6 ovariectomized female mice (SPF) which were fed with high-fat diet for 4 weeks, then they were divided into four groups, 5 animals per group. One 
group were given with estrogen along (OVCE group, $1 \mathrm{mg} / \mathrm{kg} /$ week), one group with PCSK9 inhibitor alone (OVCPi group, $3 \mathrm{mg} / \mathrm{kg} / 3 \mathrm{~d}$ ), one group with both estrogen and PCSK9 inhibitor for 2 weeks, and the last group as control. Two weeks later, all of the mice were tested as the above experiment.

Immunohistochemistry (IHC)

Paraffin section (5-Im-thick) was fixed in paraformaldehyde. The liver sections were respectively subjected to the immunohistochemical staining protocol using anti-LDLR (1:600, Abcam) and anti-PCSK9 (1:400, Abcam). Then, they were DBA color rendered and hematoxylin counterstained for analysis.

Western Blot

Total protein extracts from the mouse livers were loaded on SDS-PAGE gels for western blot. Western blot was performed according to standard protocol. The antibodies of anti-LDLR (1:3000) and anti-PCSK9 (1:3000) were used. All experiments were repeated at least three times.

Examination of PCSK9 in the blood

The content of PCSK9 were tested by ELISA(R\&D) according to the instructions of PCSK9 Elisa kit.

Lipid profiles analysis

Serum samples of the mice were analyzed with biochemical analysis kits (Nanjing Jiancheng Bioengineering Institute) to detected TC, TG, LDL-C and HDL-C.

Mouse tail genomic DNA extraction, DNA amplification and genotype identification of PCSK9-/-mouse

The Mouse tail genomic DNA were extracted by the Mouse tail genomic DNA extraction kit (APExBIO). The mouse tail DNA amplified by PCR. The genotype identification by $2 \%$ agarose gel electrophoresis. The primer sequences used for PCR were as follows (Table 1)

Table 1

Primer sequences used for PCR

\begin{tabular}{|lll|}
\hline Primer & Sequence (5'-3') & Product size (bp) \\
\hline WT-F & GCAGTCAGGAGCAGTATGTCCATGT & \\
Mut-R & TGGCTGTGAGTTGACTGTCCTTGTG & Mut: 479 \\
WT-F & GCAGTCAGGAGCAGTATGTCCATGT & WT: 540 \\
WT-R & AGAGAAGCAGAGAACGTGAGGACCC & \\
\hline
\end{tabular}




\section{Statistical analysis}

The results of WB were analyzed by ImageJ software for grayscale analysis, and then all the data were statistically analyzed using Prism 5 software. Univariate ANOVA was performed according to the paired experiment design, and $p<0.05$ was considered statistically significant.

\section{Results}

Result 1: PCSK9 increased in the blood and liver of the ovariectomized mice, as well as lipid metabolism disordered in them, and there is positive relationship between their blood PCSK9 and LDL-C.

ELISA examining mouse blood PCSK9 showed that no matter whether the ovariectomized mice were given with normal diet or high-fat diet, their blood PCSK9 increased significantly (Figure 1A. $p<0.001$, $p<0.01)$, and it increased more in those with high-fat diet (93.6\%) than those with normal diet $(77.2 \%)$ (Table 2). The examination of lipid profiles showed that the serum TC in the two groups of ovariectomized mice was both significantly increased (Figure 1.B1. $p<0.05, p<0.01$ ), and it increased in those with high-fat diet (50.4\%) more than those with normal diet (23.2\%) (Table 2); The blood LDL-c of both also increased significantly (Figure 1.B2. $p<0.01, p<0.05$ ), in the same way, the normal diet mice increased more (68.6\%) than those the high-fat diet mice (31.4\%) (Table 2). Correlation analysis showed that only LDL-C was positively correlated with PCSK9 (Figure1. D1. D2. $p<0.05, p<0.01$ ).

A. PCSK9 in the blood increased in ovariectomized mice, B1-B4. Lipid profiles in the blood; C1-C2. The correlation analysis between PCSK9 and TC under the two diets; D1-D2. The correlation analysis between PCSK9 and LDL-c under the two diets; * represents $p<0.05$, ** represents $p<0.01$, *** represents $p<0.001$

Table 2

The increased rate of PCSK9, TC and LDL-c inovariectomized mice

\begin{tabular}{|lllllll|}
\hline & Normal diet & & increased rate & High-fat diet & increased rate \\
\hline & $\begin{array}{l}\text { Sham } \\
\text { group }\end{array}$ & Ovc group & & Sham group & Ovc group & \\
PCSK9 & $132.2 \pm 17.2$ & $234.2 \pm 8.9$ & $77.2 \%$ & $194.9 \pm 13.6$ & $377.4 \pm 38.1$ & $93.6 \%$ \\
\hline TC & $2.56 \pm 0.12$ & $3.15 \pm 0.14$ & $23.2 \%$ & $3.61 \pm 0.14$ & $5.43 \pm 0.42$ & $50.4 \%$ \\
\hline LDL-C & $0.26 \pm 0.02$ & $0.44 \pm 0.04$ & $68.6 \%$ & $0.41 \pm 0.01$ & $0.54 \pm 0.04$ & $31.4 \%$ \\
\hline
\end{tabular}

Result2: PCSK9 increased and LDLR decreased in the liver of ovariectomized mice

$\mathrm{IHC}$ results showed that no matter whether the ovariectomized mice were given with normal diet or highfat diet, PCSK9 in their liver was significantly increased, while the expression of LDLR in the liver 
decreased(Figure 2.A). WB results was consistent with that of IHC (Figure 2.B1-B3, C1-C3).

Result3: There were no significant lipid disorders in PCSK $9^{-/-}$ovariectomized mice

To further prove PCSK9 in involved in lipid disorders in ovariectomized mice, PCSK9-/- mice were used for experiment. The genotype identification of the mouse was as follows (Figure 3A). Compared with the WT mice group, in which TC and LDL-c in the blood of were significantly increased in the ovariectomized mice (Figure 3.B1.B2 p<0.01, p<0.01); Compared with the PCSK $9^{-/-}$mice, there were no significant change of blood TC and LDL-C in the PCSK9 ${ }^{-/-}$ovariectomized mice (Figure 3. B1.B2). IHC results also showed that the expression of LDLR in the liver decreased in the ovariectomized WT mice, but not in the ovariectomized PCSK9-/-mice (Figure 3C). WB results were consistent with the IHC results. (Figure 3.D1.D2).

Result4: Estrogen and PCSK9 inhibitor improved the lipid disorders in ovariectomized mice.

After ovariectomized mice were treated respectively with estrogen or PCSK9 inhibitor, PCSK9, TC and LDL-C in the blood decreased significantly (Figure 4A, B1, B2, $p<0.01, p<0.05, p<0.05$ ). IHC results showed PCSK9 decreased while LDLR increased in the mouse liver (Figure 4C). WB results also showed LDLR increased in the liver(Figure 4D1,D3).; When the ovariectomized mice were treated with PCSK9 inhibitor, PCSK9, TC and LDL-C in the blood decreased(Figure 4A, B1, B2, $p<0.05, p<0.05, p<0.01$ ). IHC results showed PCSK9 decreased and LDLR increased in the liver (Figure 4C). WB results were consistent with the IHC results (Figure 4D1-D3). However, PCSK9, TC and LDL-C in the blood showed no significant differences among the ovariectomized mice treated with estrogen or PCSK9 alone or combined (Figure $4 A, B 1, B 2)$. PCSK9 and LDLR in the liver also showed no significant differences among the three group (Figure 4.C, D1-D3).

\section{Discussion}

In order to prove estrogen deficiency causing high expression PCSK9 and its relation to lipid metabolism disorders among the women in menopause, we firstly used ovariectomized mice to test their serum PCSK9 and lipid profiles. The results suggest that the ovariectomized mice had both lipid disorders and increased PCSK9, which is consistent with some result in clinical studies that is there is an increase of PCSK9 in the circulating blood of postmenopausal women [8]. Ruscica et al tested serum PCSK9 in 227 premenopausal women and 460 postmenopausal women and found that PCSK9 was significantly increased after menopause and PCSK9 was positively correlated with the degree of arteriosclerosis Related[9]. Clinical studies have shown that lipid metabolism disturbed after menopause, which is characterized by TC and LDL-c increased, accompanied by TG little increased and HDL-c decreased[2]. Thailand's clinical study of 436 subjects (152 males, 143 menopausal women and 141 postmenopausal women) showed that serum PCSK9 of female postmenopausal increased significantly and was positively correlated with TC, LDL-C and BMI. PCSK9 increased the risk of cardiovascular disease[10]. This is consistent with our results. The results of Qin 's study showed that postmenopausal women's serum 
PCSK9 increased significantly, and it was positively correlated with total cholesterol and diastolic blood pressure of age. The results showed that PCSK9 is involved in the lipid metabolism disorders in menopausal women and our result have given another more evidence.

In order to further prove the role of PCSK9 in lipid metabolism disorders in ovariectomized mice, we compared the changes in lipid profiles between WT mice and PCSK9-/-mice after ovariectomy, the results suggest that there is no obvious lipid metabolism disorder in PCSK9 ${ }^{-/-}$ovariectomized mice after ovariectomy. Clinical studies have shown that patients with PCSK9 gene deletion have increased the clearance of LDL and decreased LDL-c in plasma[11]. Jonathan C conducted a cohort study of people and found that a non-sense mutation of the PCSK9 gene led to plasma LDL-c decreased and the incidence of coronary heart disease decreased[12]. At the same time, it was also found in animal experiments that the expression of LDLR in mouse liver increased and LDL-c decreased in PCSK9 knockout mice[13]. Our previous experiments have also shown that lipid metabolism in ovariectomized mice is closely related to increased PCSK9. These studies combined with ours may prove that PCSK9 is one of the important causes of the lipid metabolism disorder in ovariectomized mice.

To investigate the effect of estrogen on PCSK9 expression, we tested the PCSK9 in the serum and liver of ovariectomized mice treated with estrogen. The results showed that PCSK9 both in the blood and liver decreased after the ovariectomized mice were treated with estrogen. Clinical studies have shown that estrogen in women is negatively correlated with PCSK9 and LDL-C[14]. Persson found High-dose ethinyl estradiol treatment in rats reduced the expression of PCSK9 while increased the expression of liver LDLR, but the mRNA level of LDLR decreased. The regulation of estrogen on liver LDLR may be completed by the post-transcriptional regulation of PCSK9[15]. All these suggest that PCSK9 is regulated by estrogen. Our results showed that when mice were ovariectomized, PCSK9 in the blood and liver increased; while when the ovariectomized mice were treated with estrogen, PCSK9 in the blood and liver decreased. All of the results indicate that the decrease of estrogen in ovariectomized mice is an important reason for the increase of PCSK9.

Above all, the increase of PCSK9 in mice ovariectomized as estrogen deficiency causes the decrease of LDLR in the liver, finally leads to disorders of lipid metabolism, which suggests that PCSK9 plays an important role in the dyslipidemia of ovariectomized mice, Therefore, PCSK9 inhibitor would be helpful for for improving the lipid metabolism disorder and reducing the incidence of cardiovascular disease in menopausal women.

\section{Declarations}

\section{Acknowledgements}

The authors gratefully acknowledge the support from the National Natural Science Foundation of China (No.81771542, http://www.nsfc.gov.cn/) and the Sichuan Natural Science Foundation, China (No.2017JY0021, URL: http://kjt.sc.gov.cn/). 


\section{Conflict of interest}

The authors have no conflict of interests.

\section{Data availability statements}

The data that support the findings of this study are available from the corresponding author upon reasonable request.

\section{References}

[1] M, S, et al, Steroid synthesis and metabolism in the nervous system: trophic and protective effects. Journal of neurocytology, 2000. 29: p 307-26.

[2] Anagnostis, $P$, et al, Effects of menopause, gender and age on lipids and high-density lipoprotein cholesterol subfractions. Maturitas, 2015. 81(1): p 62-8.

[3] Roger, V.L., et al., Heart disease and stroke statistics-2011 update: a report from the American Heart Association. Circulation, 2011. 123(4): p e18-e209.

[4] KG, J, et al, Olive oil increases the number of triacylglycerol-rich chylomicron particles compared with other oils: an effect retained when a second standard meal is fed. The American journal of clinical nutrition, 2002. 76(5): p 942-9.

[5] O'Hare, EA, et al, Disruption of Idlr causes increased LDL-c and vascular lipid accumulation in a zebrafish model of hypercholesterolemia. J Lipid Res, 2014. 55(11): p 2242-53.

[6] Wang, Y, et al, Molecular characterization of proprotein convertase subtilisin/kexin type 9-mediated degradation of the LDLR. J Lipid Res, 2012. 53(9): p 1932-43.

[7] Cui, Q, et al, Serum PCSK9 is associated with multiple metabolic factors in a large Han Chinese population. Atherosclerosis, 2010. 213(2): p 632-6.

[8] Lakoski, SG, et al, Genetic and metabolic determinants of plasma PCSK9 levels. J Clin Endocrinol Metab, 2009. 94(7): p 2537-43.

[9] Ruscica, M, et al, Circulating Levels of Proprotein Convertase Subtilisin/Kexin Type 9 and Arterial Stiffness in a Large Population Sample: Data From the Brisighella Heart Study. J Am Heart Assoc, 2017. 6(5).

[10] Jeenduang, N, Circulating PCSK9 concentrations are increased in postmenopausal women with the metabolic syndrome. Clin Chim Acta, 2019. 494: p 151-156.

[11] Cariou, B, et al, PCSK9 dominant negative mutant results in increased LDL catabolic rate and familial hypobetalipoproteinemia. Arterioscler Thromb Vasc Biol, 2009. 29(12): p 2191-7. 
[12] JC, C, et al, Sequence variations in PCSK9, low LDL, and protection against coronary heart disease. The New England journal of medicine, 2006. 354(12): p 1264-72.

[13] S, R, et al, Decreased plasma cholesterol and hypersensitivity to statins in mice lacking Pcsk9. Proceedings of the National Academy of Sciences of the United States of America, 2005. 102(15): $p$ 5374-9.

[14] Ooi, TC, et al, Relationship between testosterone, estradiol and circulating PCSK9: Cross-sectional and interventional studies in humans. Clin Chim Acta, 2015. 446: p 97-104.

[15] Persson, L, et al, Importance of proprotein convertase subtilisin/kexin type 9 in the hormonal and dietary regulation of rat liver low-density lipoprotein receptors. Endocrinology, 2009. 150(3): p 1140-6.

\section{Figures}
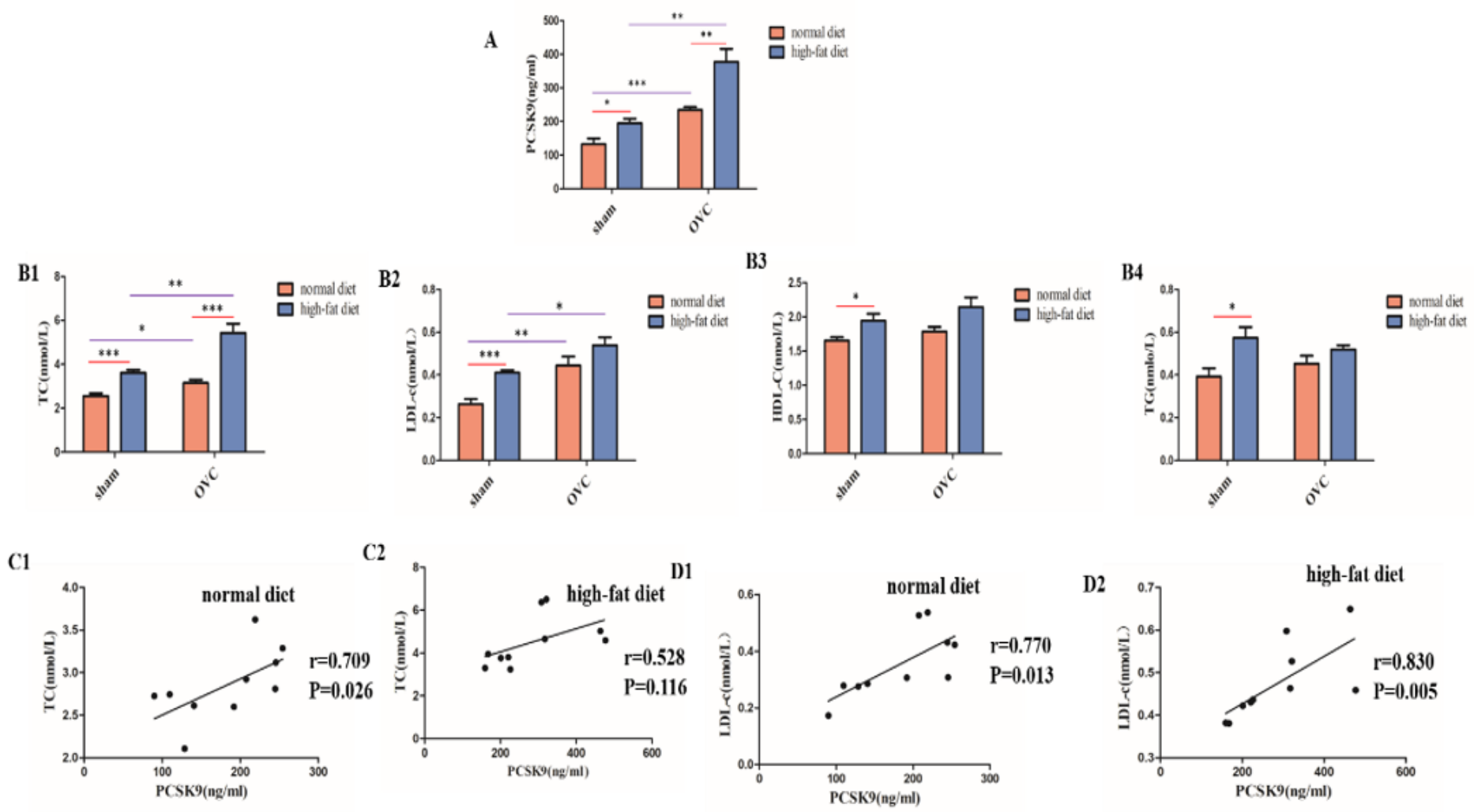

\section{Figure 1}

The change of blood PCSK9 and lipid profiles in ovariectomized mice and their correlation analysis A. PCSK9 in the blood increased in ovariectomized mice, B1-B4. Lipid profiles in the blood; C1-C2. The correlation analysis between PCSK9 and TC under the two diets; D1-D2. The correlation analysis between

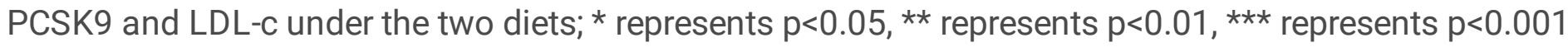




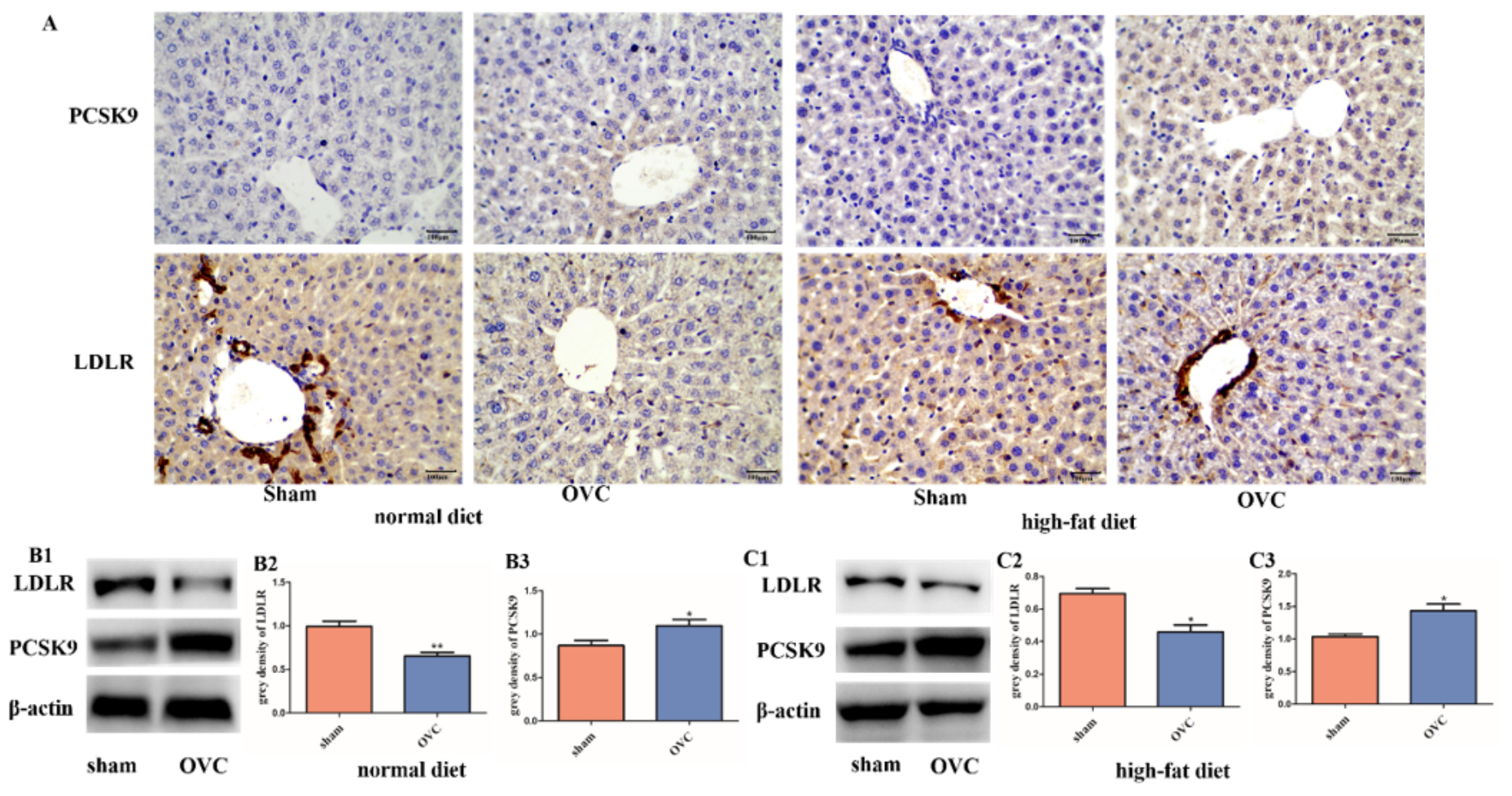

Figure 2

The change of PCSK9 and LDLR in the liver of ovariectomized mice A. The expression of PCSK9 and LDLR in the liver of ovariectomized mice by IHC, B1-B3: The expression of PCSK9 and LDLR in the liver of ovariectomized mice with normal diet by WB; C1-C3: The expression of PCSK9 and LDLR in the liver of ovariectomized mice with high-fat diet by WB; * represents $p<0.05$, ** represents $p<0.01$ 


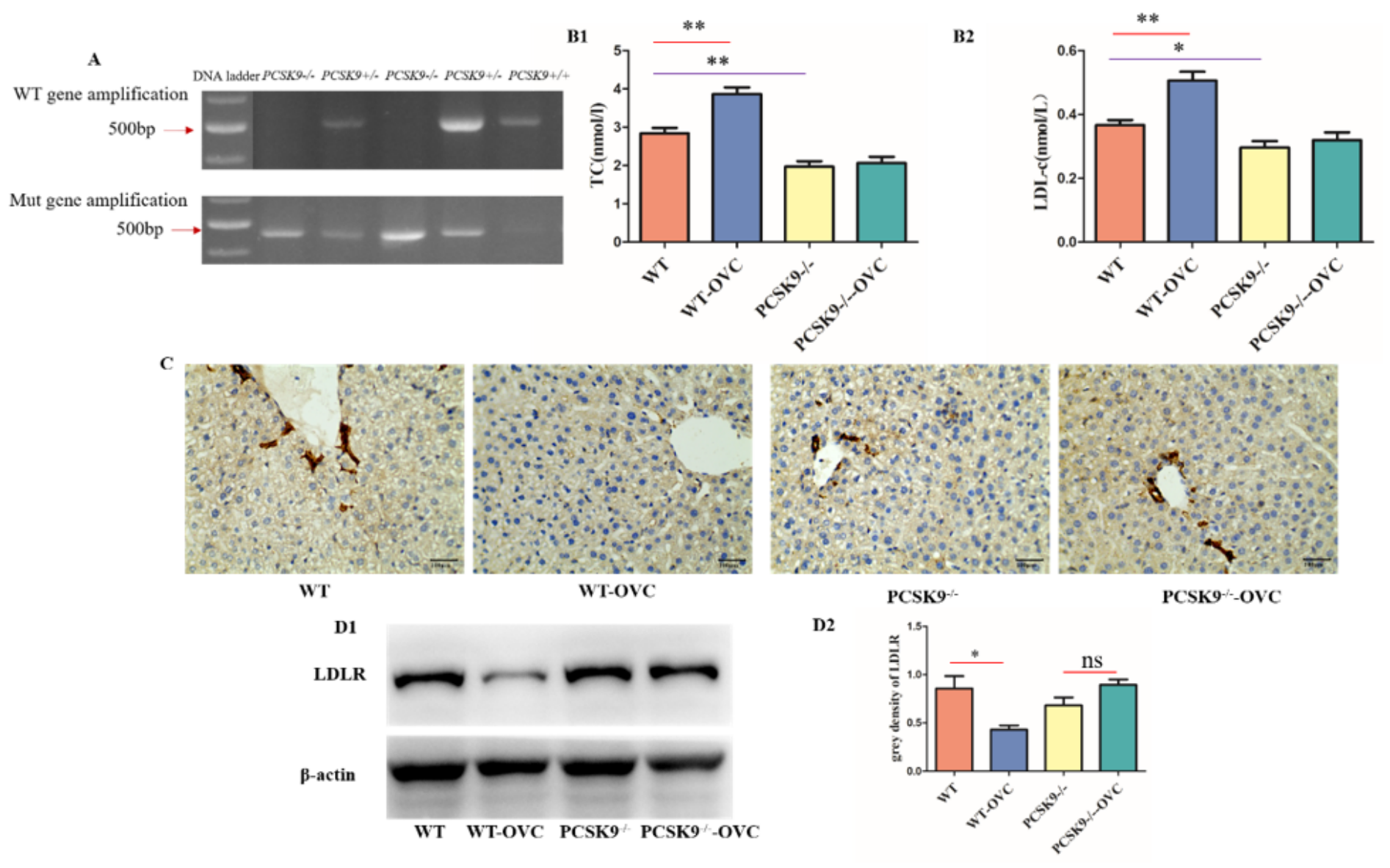

Figure 3

There were no significant lipid disorders in PCSK9 -/- ovariectomized mice A. Genotype identification of the mouse; B1-B2. TC and LDL-c in the blood; $C$. The expression of LDLR in the liver by IHC; D1-D2. The expression of LDLR in the liver by WB. * represents $p<0.05$, ** represents $p<0.01$ 


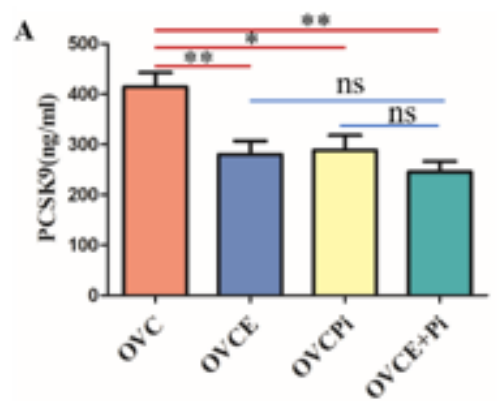

ovc
B1

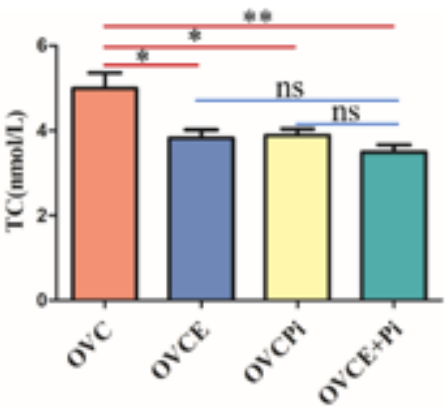

B2

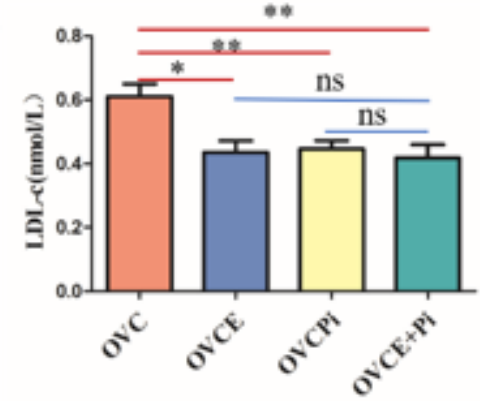

OVCE

OVCPi
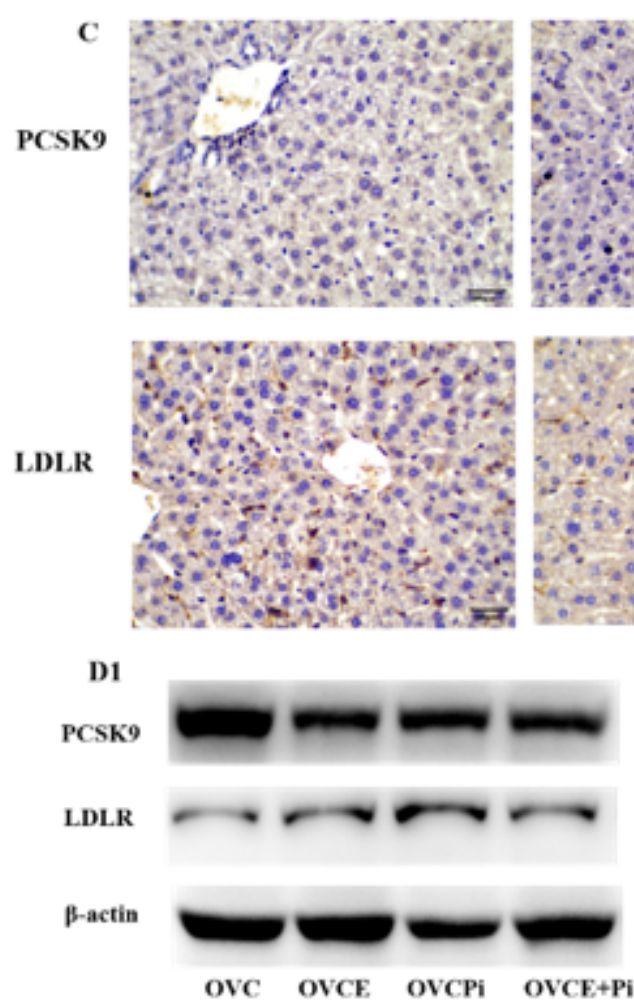
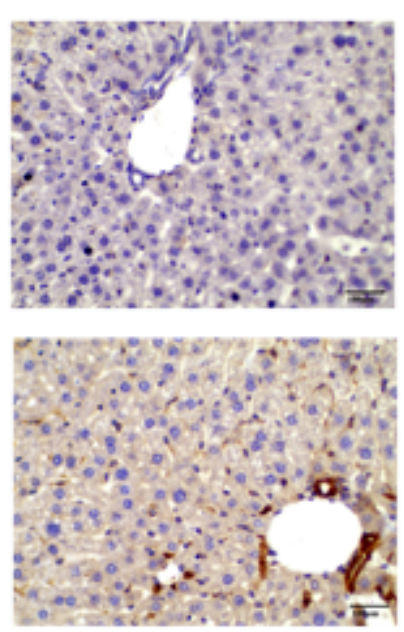

D2

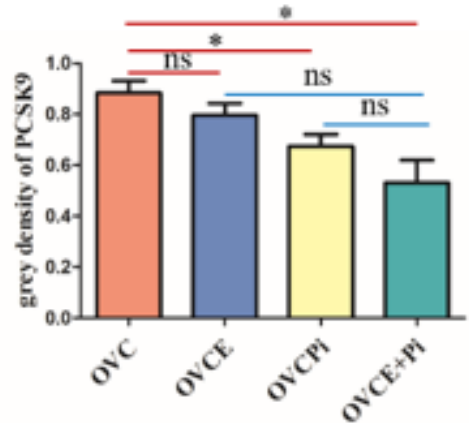

D3

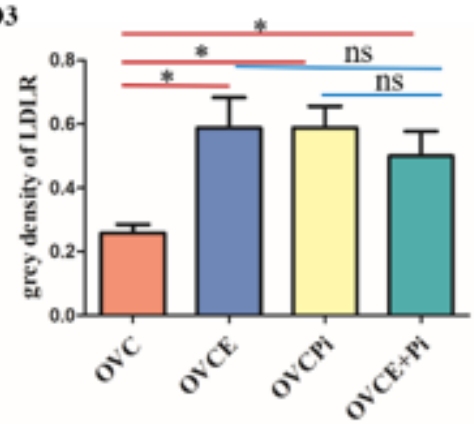

Figure 4

PCSK9 inhibitor improved the lipid disorders in ovariectomized mice A. PCSK9 in the blood of the mice; B1-B2.TC and LDL-C in the blood of the mice. C: IHC examined the expression of PCSK9 and LDLR in the liver of the ovariectomized mice treated with estrogen, PCSK9 alone or both together; D1-D3: WB examined the expression of PCSK9 and LDLR in the liver of the ovariectomized mice treated with estrogen, PCSK9 alone or both together * represents $p<0.05$, ** represents $p<0.01$ 